\title{
Advances and Limitations of Current Epigenetic Studies Investigating Mammalian Axonal Regeneration
}

\author{
Ilaria Palmisano ${ }^{1} \cdot$ Simone Di Giovanni ${ }^{1}$
}

Published online: 8 June 2018

(C) The Author(s) 2018

\begin{abstract}
Summary
Axonal regeneration relies on the expression of regenerative associated genes within a coordinated transcriptional programme, which is finely tuned as a result of the activation of several regenerative signalling pathways. In mammals, this chain of events occurs in neurons following peripheral axonal injury, however it fails upon axonal injury in the central nervous system, such as in the spinal cord and the brain. Accumulating evidence has been suggesting that epigenetic control is a key factor to initiate and sustain the regenerative transcriptional response and that it might contribute to regenerative success versus failure. This review will discuss experimental evidence so far showing a role for epigenetic regulation in models of peripheral and central nervous system axonal injury. It will also propose future directions to fill key knowledge gaps and to test whether epigenetic control might indeed discriminate between regenerative success and failure.
\end{abstract}

Keywords Epigenetic $\cdot$ HDAC $\cdot$ HAT $\cdot$ Transcription · Axonal regeneration · Spinal cord injury $\cdot$ Nerve injury $\cdot$ DNA methylation $\cdot$ RNA

\section{Introduction}

Injury to the nervous system, such as following traumatic spinal cord and brain injuries, or a vascular insult such as stroke, causes loss of neuronal function leading to sensory, motor, autonomic or cognitive impairment. Successful compensatory axonal sprouting from intact neurons or regeneration from injured axons, i.e. regrowth of the injured axon to the original target is required for functional recovery. In mammals, axonal regeneration relies on the synergy between the presence of a permissive extracellular environment and the intrinsic growth capacity of the injured neurons [1-3]. While the mammalian peripheral nervous system (PNS) axons are able to regenerate to a certain extent, axons in the central nervous system (CNS) do not, due to the presence of glial inhibitory signalling, glial scar and to a limited neuronal

Ilaria Palmisano

i.palmisano@imperial.ac.uk

Simone Di Giovanni

s.di-giovanni@imperial.ac.uk

1 Laboratory for Neuroregeneration, Centre for Restorative Neuroscience, Division of Brain Sciences, Department of Medicine, Imperial College London, London, UK intrinsic regenerative ability [4-6]. Interference with the inhibitory glial extra-neuronal cues has resulted into an improvement of axonal regrowth in the injured CNS [7-9]. However, this remains rather limited, and it has prompted the development of strategies aimed to enhance the poor neuronal intrinsic growth ability. While protein translation seems to play a role (reviewed elsewhere $[10,11])$, the axon growth competence of neurons in both the PNS and CNS depends mainly on gene transcription, and on the capability to initiate an orchestrated transcriptional response to injury [12]. This ability declines during neuronal maturation and with aging [13-17]. The decline in regenerative ability is mainly due to changes in the transcriptional programme, which is affected by an overall reduction in chromatin accessibility at gene regulatory regions as a function of development. This is associated with a drop in the level of transcription factors (TFs) and in the transcription of genes needed for axonal regeneration $[18,19]$, and it might explain why forced expression of TFs or regeneration associated genes (RAGs) [20], alone or in combination, has resulted in limited axonal regeneration so far [21-23]. Although some experiments are biased by the heterogeneity of the nervous system tissue, there is evidence that expression of epigenetic modifiers and epigenetic marks are regulated during development. For example, immunoblotting of $\mathrm{H} 3 \mathrm{~K} 9 / 14 \mathrm{ac}$ from cortical and cerebellar granule neuron 
(CGN) extracts [24] as well as H3K18ac and p300 immunostaining in retinal ganglia neurons (RGNs) [25] has revealed a decrease in the expression of these positive epigenetic marks in mature compared to earlier developmental stages. A chromatin immunoprecipitation small screening of histone posttranslational modifications (HPTM) in cortical neurons revealed pronounced changes in histone marks at the promoter regions of several RAGs, indicative of progressive reduced chromatin accessibility during post-natal development [19].

Given the importance of chromatin structure for the accessibility of TFs to gene promoters and other gene regulatory elements, epigenetic mechanisms that control chromatin remodelling are strong candidates to play a crucial role in axonal regeneration. Here we provide a critical discussion of the role of epigenetic-dependent transcriptional mechanisms in mammalian axonal injury and we suggest future research directions.

\section{Epigenetic Mechanisms}

Epigenetic modifications underpin changes in gene expression without alteration of the DNA sequence. In eukaryotes, DNA is organised in a hierarchical structure called chromatin, where its first order organisation is determined by the wrapping of DNA around histone proteins into nucleosomes, while the secondary and tertiary structures are stabilized and maintained by architectural proteins and chromatin remodelling factors [26, 27]. The degree of chromatin compaction plays a critical role in gene regulation, with highly transcribed genes and active regulatory elements, such as promoters and enhancers, being associated with a more relaxed and more accessible chromatin configuration [26-28]. The dynamic equilibrium between compact vs relaxed chromatin state in response to different stimuli is the result of multiple mechanisms, such as DNA methylation and hydroxymethylation, various HPTMs, exchange of histone variants, binding or displacement of architectural proteins, including ATP-dependent remodelling factors [29-31]. Nucleosomes are formed by the wrapping of $147 \mathrm{bp}$ of DNA around a histone octamer, consisting of 2 units each of $\mathrm{H} 2 \mathrm{~A}, \mathrm{H} 2 \mathrm{~B}, \mathrm{H} 3$ and $\mathrm{H} 4$, stabilized by the association with $\mathrm{H} 1$ linker histone [32]. The presence of nucleosomes generally inhibits transcription, due to DNA bending or steric hindrance reducing the availability for transcription machinery to DNA binding [33, 34]. However, chromatin compaction and accessibility can be modified by the modifications of specific residues at the N-terminal tail of histones, affecting their interaction with the DNA. These modifications, including acetylation, methylation, phosphorylation, ubiquitination and SUMOylation, are the result of the balance between chromatin erasers and writers, which are enzymes responsible for removal or addition of such modifications, respectively, as reviewed elsewhere [35]. According to the so-called 'histone code' hypothesis, while histone modifications affect chromatin conformation, they can also indirectly exert their influence on gene transcription, via the recruitment of specific chromatin readers.

Acetylation at the $\varepsilon$-amino group of specific lysine $(\mathrm{K})$ residues is the best characterized HPTM, and it is generally associated with transcriptional activation, providing binding sites for bromodomain proteins [36]. It is regulated by the balance between the activity of histone acetyl transferases (HATs) and histone deacetylases (HDACs) [35, 37]. HATs belong to three major families: general control nonderepressible 5 (Gcn5)-related N-acetyltransferases (GNATs), p300/CREB binding protein (CBP), and MYST [35]. The GNAT family includes the p300/CBP associated factor (PCAF). HDACs belong to four classes: class I-IV. All HDACs are zinc-dependent enzymes, except for class III HDACs, or sirtuins, which are $\mathrm{NAD}^{+}$-dependent enzymes, and constitute a structurally separate family [35]. Class I HDACs are ubiquitous proteins with high deacetylase activity toward histones, while class II HDACs display tissue expression specificity, prominent nucleo-cytoplasmic shuttling and a weak enzymatic activity, which in vivo is given by their association to class I HDACs [38].

Another level of epigenetic control is determined by DNA methylation. DNA methylation involves the covalent transfer of a methyl group to the C-5 position of the cytosine by DNA methyltransferases (DNMTs) [31, 39]. DNA methylation is regulated by a family of DNMTs: DNMT1, DNMT2, DNMT3A, DNMT3B, and DNMT3L [40]. While DNMT1 is generally involved in maintenance of methylation, copying DNA methylation patterns to the daughter strand during DNA replication [41], DNMT3A, DNMT3B, with the assistance of DNMT3L, are mainly responsible for de novo DNA methylation [42]. In mammals, more than $98 \%$ of DNA methylation occurs on $\mathrm{CpG}$ dinucleotides in somatic cells, while non-CpG methylation is more abundant in embryonic stem cells and brain tissue, where neurons show higher levels than glial cells $[43,44]$. The $\mathrm{CpG}$ methylation pattern is found throughout the genome, with the exception of short unmethylated regions called $\mathrm{CpG}$ islands, mostly coinciding with promoters and first exons of protein coding genes [45-47]. Conventionally, methylation of DNA exerts a repressive role on gene transcription, acting at different levels. It represents a physical impediment to the binding of TFs, and more importantly, it is a docking point for methyl $\mathrm{CpG}$ binding domain (MBD) family proteins. These proteins are able to interact with a large variety of other factors, including HDACs, histone methyltransferases, polycomb complexes, and ATP-dependent chromatin remodelling factors, altering the histone code, influencing nucleosome stability and positioning as well as chromatin high order structure [31, 47, 48].

DNA demethylation has long been thought to be a passive event associated to the loss of 5-methylcytosine $(5 \mathrm{mC})$ during 
successive rounds of replication, whereas active removal of the methyl group from $5 \mathrm{mC}$ has been controversial until the discovery of the ten eleven translocation (TET) enzymes, TET1, TET2, and TET3 [49-51]. TET enzymes catalyse the consecutive oxidation of $5 \mathrm{mC} 5$-methylcytosine $(5 \mathrm{mC})$ to 5 hydroxymethylcytosine $(5 \mathrm{hmC}), 5$-formylcytosine $(5 \mathrm{fC})$, and 5 -carboxylcytosine $(5 \mathrm{caC})$ [52]. The thymine DNA glycosylase (TDG) can subsequently excise TET oxidation products, leading to the complete DNA demethylation [51]. While $5 \mathrm{mC}$ is found in all mammalian tissues, corresponding to almost $4-5 \%$ of all cytosines, $5 \mathrm{hmC}$ level is lower and displays a tissue specific pattern. It is prevalent in embryonic stem cells and in the brain where it can reach about $40 \%$ of the total $5 \mathrm{mC}$, while it is rare in the spleen and testes (only about $0.03 \%-0.06 \%$ ) [49]. Nevertheless, this modification, along with $5 \mathrm{fc}$ and $5 \mathrm{ca}$, is thought not to be a mere transient intermediate of the DNA demethylation enzymatic process but to be persistent in time and to facilitate gene expression [53]. Interestingly, in the mouse and human brain, $5 \mathrm{hmC}$ is enriched at genes involved in synaptic function [54].

\section{Histone Acetylation: HDAC Inhibitors, HATs and HDACs in Axonal Injury and Regeneration}

\section{HDAC Inhibitors}

The importance of histone acetylation in axonal regeneration has initially been suggested by experiments with broad range HDAC inhibitors (HDACi), such as pan-HDACi trichostatin A (TSA) in cultured rat CGN. TSA increased acetylation of $\mathrm{H} 3$ at $\mathrm{K} 9 / 14$, neurite extension and growth cone remodelling and this required transcription, since transcriptional inhibitors blocked TSA-dependent neurite outgrowth [24]. The effect was observed on both growth permissive and nonpermissive substrates such as myelin, in line with other evidence showing that treatment with class I and II HDACi Valproic acid (VPA) allowed cultured embryonic spinal cord and hippocampus neurons to partially overcome growth inhibition by the myelin associated protein NogoA. Interestingly, VPA enhanced recovery of locomotion in rat spinal cord contusion model, however the mechanisms underlying this recovery had not been explored $[55,56]$. Subsequently, it has been reported that TSA injection into the vitreous at the time of an optic nerve crush (ONC) in rats increased RGN survival and H3K18ac levels, although it failed to enhance axonal regeneration [25]. Systemic injection of TSA or of class I HDAC inhibitor MS-275 led to a global increase of acH4 in dorsal root ganglia (DRG) neurons and specifically at the promoters of several RAGs. This also resulted in increased RAG expression and in enhanced neurite outgrowth of ex vivo cultured DRG neurons [57]. Since the positive effect on neurite outgrowth was observed up to 2 weeks after the last injection of
MS-275, when the level of histone acetylation at the RAG's promoters had come back to baseline, this suggests a longlasting increase in acetylation on other target genes or nonhistone acetylation dependent mechanisms. However, the in vivo treatment with MS-275 limited axonal die-back but did not result in significant axonal regeneration of DRG sensory fibers beyond the spinal injury site [57]. Despite the lack of selectivity towards individual HDACs and/or to the lack of cellular specificity, these findings suggested that the inhibition of HDACs leading to an increase in histone acetylation might be beneficial for axonal regeneration. Indeed, using DRG pseudo-unipolar neurons as a model to compare molecular responses between a regenerative peripheral vs nonregenerative central axonal injury [58] within the same cell body, it has been found that acH4 level increased $6 \mathrm{~h}$ after a peripheral, but not a central axonal injury. This occurred both globally in DRG neurons and on the promoters of a few tested RAGs [57]. Accordingly, in a low throughput screening of HPTMs 24 hours following axonal injury, Puttagunta et al. [59] showed that H3K9ac level increased upon sciatic nerve axotomy (SNA) in DRG neurons together with H3K9ac occupancy on the promoter of selected RAGs, such as Galanin, Gap43, and Bdnf. On the contrary, H3K9ac levels decreased upon dorsal column axotomy (DCA), while the repressive mark H3K9me2 was enriched on the same promoters. These changes correlated with gene expression, although not for all of the tested RAGs. These studies suggested the presence of a differential chromatin state upon these two types of injury and that the increase of each histone mark is only partially correlated to the activation of the entire transcriptional programme, pointing to the need for more systematic high throughput studies.

\section{HATs}

Several studies have identified CBP/p300 and PCAF as HATs that play important roles in neurite outgrowth and axonal regeneration. Using small interfering RNA (siRNA) approaches Gaub et al. [24] identified CBP/p300 and PCAF as responsible for TSA-induced H3K9/14ac and neurite outgrowth in CGN. Neurite growth in control cells was not significantly affected on permissive substrates, suggesting a compensation between these enzymes, or that a minimal level of expression might suffice for neurite outgrowth in permissive growth conditions. Q-PCR and ChIP-qPCR experiments in TSA treated CGN indicated that TSA induces increased $\mathrm{CBP} / \mathrm{p} 300$ and PCAF expression correlating with higher $\mathrm{H} 3$ acetylation level on their promoters. Although those experiments had the bias of glial cell contamination, the result was supported by the correlation between single cell $\mathrm{CBP} / \mathrm{p} 300$ expression, $\mathrm{H} 3$ acetylation level, and neurite outgrowth in TSA treated cells. A similar analysis in control vs treated cells, and qPCR from a pure neuronal population would have helped clarify whether TSA is actually responsible for the increased expression of 
$\mathrm{CBP} / \mathrm{p} 300$ and PCAF via increased histone acetylation at their promoters. However, overexpression of $\mathrm{CBP} / \mathrm{p} 300$ and $\mathrm{PCAF}$ in CGN by electroporation was sufficient to enhance neurite outgrowth in permissive and inhibitory substrate to an extent comparable to TSA treatment.

In follow up studies Gaub et al. [25] showed that the failure of TSA in promoting axonal regeneration following ONC correlated with the lack of induction of p300 expression. Accordingly, adeno-associated virus (AAV) mediated p300 overexpression in the eye, via intravitreal injection, was sufficient to increase axonal regeneration in RGN after ONC, synergising also with lens injury, a conditioning lesion that enhances optic nerve axonal regeneration via inflammatory stimulation. Overexpression of p300 increased H3K18ac, the occupancy of p300 and acH3 on the promoter of growthassociated genes such as Gap43, Sprrla, Coronin 1b that also displayed increased gene expression. P300 overexpression in RGN likely underpins cell autonomous effects upon axonal regeneration, although expression in amacrine and bipolar neurons was observed too.

In agreement with these findings [24], in vitro AAV mediated PCAF overexpression resulted in increased neurite growth on permissive and inhibitory environment on both cultured DRG and CGN. This led to increased expression of selected RAGs, while the PCAF inhibitor Garcinol inhibited neurite outgrowth and regenerative gene expression [59]. Remarkably, a single Garcinol intrathecal injection just before SNA, reversed the conditioning-dependent increase in DRG neurite outgrowth. Since Garcinol can also inhibit p300/CBP, which are involved in axonal regeneration [25], the authors confirmed the specificity of these results by using PCAF null mice. DRG neurons from PCAF null mice failed to display regenerative growth both in culture and in vivo after a conditioning lesion and spinal cord injury, although the use of a neuronal specific PCAF knock out (KO) would have been advisable to distinguish between neuronal vs non-neuronal specific effects. More importantly, in vivo PCAF overexpression in DRG neurons mediated by AAV was sufficient alone to induce increased level of $\mathrm{H} 3 \mathrm{~K} 9 \mathrm{ac}$ and increased expression of RAGs. PCAF overexpression promoted sensory axonal regeneration up to $1 \mathrm{~mm}$ from the lesion site in a T9-T10 spinal dorsal column crush, mimicking and surpassing the regenerative ability of a conditioning lesion. Although it is difficult to compare different studies because of different spinal cord injury (SCI) models, and despite functional tests have not been performed, it is worth noting that the degree of axonal regeneration achieved in this study was higher than the one obtained with MS-275 injection after a C5 dorsal column axotomy [57]. This possibly reflects the fact that MS-275 was able to mimic the regenerative transcriptional programme only partially but it also suggests that epigenetic changes on key genes, working as functional hubs in neurons [60], may be better able to elicit axonal regeneration compared to global, less specific, changes driven by HDACi. In this regard, high throughput studies will be necessary to fully characterize the role of PCAF on gene promoters and enhancers.

An interesting biological question is how HATs are modulated after axonal injury. Puttagunta et al. demonstrated for the first time a link between retrogradely transported signalling molecules and epigenetic modifications [59]. They showed that activation of PCAF and its binding to RAG promoters was ERK dependent, whose phosphorylation and retrograde transport increased following injury [61, 62]. Indeed, nerve treatment with a MEK1/2 inhibitor abolished the SNA dependent increase in the phosphorylation of PCAF, H3K9ac level globally in DRG nuclei and specifically on the RAG promoters. It also abolished ex vivo neurite outgrowth, whereas NGF treatment, activating pERK signalling, mimicked the injury dependent increase in PCAF phosphorylation.

\section{HDACs}

The very first molecular events following a peripheral axonal injury are represented by changes in electrical activity and $\mathrm{Ca}^{++}$influx $[63,64]$ that lead to an increase in cAMP levels $[65,66]$ and activation of multiple signalling cascades $[2,62$, 67, 68]. Cho et al. [69] showed that a peripheral axotomy induced the nuclear export of HDAC5 towards the periphery in DRG. This relied upon $\mathrm{Ca}^{++}$, protein kinase $\mathrm{C}(\mathrm{PKC})$ and microtubule cytoskeleton. HDAC5 export increased tubulin deacetylation and acH3 level in the nuclei of DRG neurons, contributing to growth cone dynamics and axonal regeneration [70]. Taking advantage of a mutant form of HDAC5 that cannot be transported but it is entrapped in the nucleus (HDAC5nuc), they demonstrated that the HDAC5 nuclear export is needed for axonal regeneration in culture and for the expression of a regenerative transcriptional programme. Indeed a gene microarray study at 3-8-12 h upon in vitro axotomy in GFP and HDAC5nuc expressing DRG neurons identified injury responsive genes dependent from HDAC5 nuclear export. While injury responsive genes were more abundant at $8 \mathrm{~h}$ upon axotomy, the ones dependent from HDAC5 were more represented at 3 and $12 \mathrm{~h}$, reaching about $50 \%$ of the transcriptional programme, pointing to a role for HDCA5 in controlling early response genes. HDAC5dependent gene expression included functional categories related to transcription, cell adhesion, and signalling. In line with this, subcutaneous injection of I3A, an activator of $\mathrm{PKC}$, was able to mimic and synergize upon DRG regenerative growth with the sciatic nerve conditioning lesion in $e x$ vivo cultured DRG. I3A delivery was also able to enhance HDAC5-dependent axonal regeneration in vivo upon nerve crush. Since class II HDAC enzymatic activity is low compared to other HDACs, and HDAC4 and HDAC5 have been shown to interact with HDAC3 [38, 71], the authors confirmed that HDAC5 controls the subcellular distribution of 
HDAC3 and its nuclear export upon axonal injury in DRG neurons. Therefore, further studies will be necessary to distinguish between HDAC5 direct vs indirect, possibly via HDAC3, effects on gene expression. This work proposes HDAC5 and HDAC3 as interesting candidates to be tested in models of CNS injury, where HDAC5 and HDAC3 nuclear export may fail to happen, as suggested by studies in RGN $[70,72]$. Recently, HDAC3 inhibition has been shown to have beneficial effect on functional recovery and myelination upon SCI and nerve injury, acting on the polarization of microglia/ macrophages and Schwann cells respectively [73, 74]. This does not exclude additional roles in neurons, since a neuroprotective role of HDAC3 inhibition has been proven in RGN $[75,76]$. Similarly, combined genetic ablation of Hdacl and 2 has recently been shown to promote neuroprotection following optic nerve axotomy via p53-dependent pathways [77]. HDAC2 genetic or pharmacological inhibition has also been found to promote recovery of motor function, to enhance cell survival and neuroplasticity as well as to reduce neuroinflammation in a stroke model in rodents $[78,79]$.

\section{Non-histone Acetylation in Axonal Injury and Regeneration}

HATs and HDACs can have additional targets other than histone proteins [80], and some of those non-histone acetylation events have been linked to axonal regeneration. For example, p300/CBP and PCAF can mediate axonal regeneration also via acetylation of $\mathrm{p} 53$ on $\mathrm{K} 373 / 320$ and $\mathrm{C} / \mathrm{EBP}[24,25,81]$. However, further studies are needed to discriminate between histone acetylation vs non-histone acetylation contribution of p300 or TSA mediated axonal regeneration. The data seems to imply that in these paradigms the contribution of the acetylation of p53, C/EBP or of other TFs might overcome histone acetylation-dependent axonal regeneration since (i) the overexpression of a mutant form of p53 that cannot be acetylated almost completely abolished neurite outgrowth in control and TSA treated CGN; (ii) TSA treatment, which did not affect acetylation of $\mathrm{p} 53$, failed to induce axonal regeneration. Lastly, active acetylated p53, following MDM2/4 inhibition, enhanced axonal sprouting and regeneration following spinal cord injury irrespective of histone acetylation [82].

Finelli et al. [57] described a positive feedback between histone acetylation and the function of SMAD1, a TF previously identified to be involved in axonal elongation [83]. They found an inverse correlation between pSMAD1 occupancy and HDAC1 binding at four gene promoters (Sprrla, Galanin, Vip, Npy) and a positive correlation with p300 binding at Sprrla promoter in Neuro2A cell lines. Moreover, pSMAD1 was found to interact with p300 in a lysate of cultured DRG. These studies have the limitations to have been performed in Neuro2A cell lines or in cultured DRGs, therefore whether such a complex on DRG derived chromatin exists in vivo remains unclear and it deserves further investigations. Nevertheless, it suggests that SMAD1, similarly to SMAD2 and SMAD3 [84], can be a target of p300-dependent acetylation.

Injury dependent cytoplasmic export of HDAC5 led to decreased level of acetylated tubulin, which correlated with microtubule stability. In this way, HDAC5 controls microtubule dynamics in injured DRG neurons, improving growth cone remodelling and regeneration [69]. Other studies indicated that HDAC6 inhibition too can mediate both neuroprotection and axon growth on myelin substrate via $\alpha$-tubulin acetylation [85]. This apparent contradictory result can be explained by the different experimental conditions including an axotomy in the HDAC5 study and growth on inhibitory substrates for HDAC6. Additionally, HDAC6 is known to deacetylate, among other proteins, cortactin, which is required for actin and growth cone remodelling [85-87].

Together, non-histone acetylation deserves further investigation, since acetylation/deacetylation of several TFs or signalling molecules, such as STAT3, c-myc, SMAD7, NF-Kb, HIF1a, FOXO1, E2F, phosphatase and tensin homolog (PTEN), many of which are involved in axonal regeneration, has been shown to affect their stability and/or their activity [80, 88, 89], thus potentially impacting the axonal regenerative ability.

\section{DNA Methylation and Hydroxymethylation in Axonal Injury and Regeneration}

Using a combined spinal cord and conditioning sciatic nerve injury in rats, it was found that axonal injury induces increased expression of the folate surface receptor 1 (FLR1), as shown by in situ hybridization and immunohistochemistry in DRG cell bodies and by qPCR in the spinal cord [90]. FLR1 was required for DRG neurite outgrowth in culture, since DRG neurite outgrowth was significantly impaired in $\mathrm{Flr}^{+/}$mice. Moreover, folate supplementation was able to promote axonal regeneration in CNS injury models, such as optic nerve and dorsal column cervical transection, however this did not occur in $\mathrm{Flr} 1^{+/-}$mice. Since axonal injury was accompanied by decreased DNMT3a and DNMT3b protein levels and decreased DNA methylation in the spinal cord, folate supplementation seems to counteract the injury-dependent hypomethylation, by restoring the level of DNMT3a and DNMT3b. Indeed, folate supplementation affected global methylation levels and specific methylation of the Gadd45a promoter in the spinal cord, and the DNMT antagonist 5-azacytidine (5-AzaC) partially blocked folate-dependent spinal axonal regeneration in vivo [90]. However, since most of the assessments were carried out in bulk spinal cord tissue, it remains to be clarified whether this reflects an effect on neuronal or glial cells. Use of neuronal specific Flr1 knock down (KD) mouse models and/ or cell specific assessment of the DNA methylation and 
DNMT levels would have been helpful in this regard. The consequence of folate intake on the levels of DNA methylation, and expression of DNMTs and MDMB proteins is well established. Although the mechanisms are still unclear, it is one of the interesting examples of how nutrition/diet may affect the epigenome [91, 92]. A later study [93] showed the cell specific expression of DNMTs in rat DRG neurons by immunohistochemical analysis. While DNMT1 was expressed in both glia and neurons, DNMT3a was preferentially expressed in glia and DNMT3b was preferentially expressed in neurons, although the use of specific glial and neuronal subtype markers would have complemented the analysis to identify possible different expression patterns among glial and neuronal cell types. Using a nerve ligation model of neuropathic pain, qPCR studies revealed a robust increase of DNMT3a expression only at one week upon injury, which was sustained up to four weeks. However, DNMT1 and DNMT3b increase was weaker and delayed weeks after ligation. These studies seem at odds with earlier investigations revealing that DNMT1 and DNMT3a were highly expressed in adult post-mitotic neurons, whereas DNMT3b was poorly expressed [94-96]. However, together with Iskandar et al. [90], they indicate that DNA methylation could be affected following axonal injury.

The first attempt to answer this question with a high throughput approach, checking for promoter and $\mathrm{CpG}$ island specific changes, was a microarray study performed comparing DNA methylation pattern in mouse DRG at 1, 3, and 7 days upon a peripheral sciatic nerve axotomy or a central dorsal column spinal axotomy $[59,97]$. Although only a modest number of genes were found to be differentially methylated with respect to sham injury and none was associated to RAGs, the methylation temporal dynamics was distinct in the two conditions. Hypermethylated genes were prevalent at 1 day after SNA, while hypomethylated genes peaked at 7 days upon DCA. More interestingly, differentially methylated genes related to transcription and chromatin remodelling were well-represented upon SNA but they were hardly found among hypomethylated genes following DCA. Following nerve axotomy, ion channels were clearly more abundant among hypermethylated genes with respect to hypomethylated, and this is interesting since ion channels have been found to be regulated following injury and linked to axonal extension and regeneration [98-101]. Nevertheless, this study has the limitation to be biased for promoters and $\mathrm{CpG}$ islands of the 18,000 genes represented in the arrays, and the whole DRG used contained both neurons and glial cells. Further genome wide studies are necessary to characterize the DRG DNA methylome upon axonal injury, possibly at the single cell level.

Recently, $5 \mathrm{hmC}$ has deserved much attention in cell biology. Both Loh et al. and Weng et al. [102, 103] found that peripheral nerve injury results in increased level of TET3, but not TET1 and TET2, and 5hmC in DRG. TET3 increase was counteracted by the use of $\mathrm{Ca}^{++}$chelators [102], suggesting that injury-dependent increase in calcium signaling might be required for $5 \mathrm{hmC}$. Moreover, TET3 KD by AAV mediated short hairpin (shRNA) abolished nerve injury dependent increase of $5 \mathrm{hmC}$ [102]. Using a next generation sequencing (NGS) approach in DRGs following $5 \mathrm{hmC}$ capture, Loh et al. showed changes in $5 \mathrm{hmC}$ pattern at unique genomic locations upon peripheral and central axotomy with respect to naïve tissue [103]. Surprisingly, a higher number of differentially hydroxymethylated regions (DhMRs) upon DCA, with a bias for a gain in $5 \mathrm{hmC}$, with respect to SNA was described. About $40 \%$ of the RAGs previously identified by the same group by microarrays [83] presented differential hydroxymethylation, but a clear correlation with their expression was not evident. This poor correlation might be affected by the limitation of comparing microarrays vs next-generation sequencing studies. Additionally, approximately half of DhMRassociated RAGs contain multiple DhMRs, often displaying opposite patterns and the majority of the DhMR occurred at 'open sea' regions. Interestingly, signalling pathways and TF motif analysis of the peripheral and central DhMRs revealed association to different repertoires of signalling molecules and TFs. For example, IRF3/5 and STAT families were specifically associated to peripheral DhMRs, ARI3A, FOX, and MAZ were specifically associated to the central DhMRs, whereas HIF1A and ARNT were present in both. Some of those TFs have been associated to transcriptional response to axonal injury and/or axonal regeneration [104-106], whereas others, such as FOX and ARI3A, are interesting candidates for further studies, since they have been linked to chromatin remodelling [107-109].

In line with these findings, Weng et al. [102] found that TET3 KD by AAV mediated shRNA reduced the number and length of DRG neurites in culture and nerve regeneration in vivo. This was not due to cell loss as demonstrated by caspase immunostaining. While WT animals showed signs of skin reinnervation and progressive functional recovery at the heatevoked hind paw withdrawal test, TET3 KD mice showed significantly impaired responses. Q-PCR and immunohistochemistry analysis revealed that TET3 KD attenuated the nerve injury induced expression of several RAGs, such as ATF3, STAT3, SMAD1, and c-myc, with SMAD1 and STAT3 expression being impaired also in naïve condition. Performing targeted bisulfite sequencing from a neuronal purified preparation the authors proved that nerve injury induced TET3 occupancy and DNA demethylation at enhancers and gene bodies of specific RAGs, and that this was lost in TET3 shRNA transduced neurons. Interestingly, in a model of regenerating CNS axons following PTEN deletion in RGNs [110], TET1 was required for nerve regeneration. Further studies are needed to address the regulation of TET proteins upon axonal injury. Little is known about post-translational modifications of TET enzymes. They can be phosphorylated by JNK, PKC, and ATM resulting in changes in their cellular 
localization, protein stability and function. Phosphorylation can be suppressed via $O$-GlcNAcylation by the glycosyltransferase OGT $[111,112]$. Interestingly, TET proteins, by recruiting OGT to chromatin, can also participate in $O$ GlcNAc modification of histones, a newly discovered HPTM linking nutrient availability to chromatin remodelling [113]. Another outstanding question that needs to be addressed is the contribution of the loss of DNA methylation vs the increase of DNA $5 \mathrm{hmC}$ in axonal regeneration. Weng et al. [102] found that the KD or KO of TDG, the enzyme responsible for the removal of TET oxidation products and complete DNA demethylation [51], impaired nerve regeneration and RAG expression in a similar way compared to TET3 KD. Intriguingly, TDG KD led to enhanced levels of $5 \mathrm{hmC}$, suggesting that increased $5 \mathrm{hmC}$ level may not be sufficient for RAG expression and subsequent axonal regeneration, but rather that complete DNA demethylation must occur. This might also partially explain the lack of consistent correlation between $5 \mathrm{hmC}$ changes and RAG expression in the Loh study [103]. It would be therefore interestingly to correlate $5 \mathrm{hmC}$ changes with DNA methylation levels during a time course upon axonal injury. This would allow evaluating whether a higher association with gene expression can be found for genes showing DNA demethylation following an increase in $5 \mathrm{hmC}$.

\section{RNA Mediated Regulation in Axonal Injury and Regeneration}

Several studies have uncovered the role of non-protein coding RNA transcripts, such as microRNAs (miRNAs), small interfering RNAs such as microRNAs (miRNAs), siRNAs, Piwiinteracting RNAs (piRNAs), and long non-coding RNAs (lncRNAs), in the control of gene regulation via epigenetic mechanisms. LncRNAs mediate gene silencing by recruiting Polycomb proteins, histone methyltransferases, and DNMTs, and they may display enhancer like function [114]. Many miRNAs can control gene expression by affecting expression of epigenetic remodellers, such as Polycomb proteins, HDACs, DNMT3a, p300. There is evidence that miRNAs and lncRNAs are differentially expressed in the neural tissue after various types of injury, such as nerve, spinal cord, traumatic and ischemic brain injury, where they promote regeneration by regulating key biological processes, including cell survival/apoptosis, extracellular matrix-cytoskeleton signalling, and neurite outgrowth [115]. Therefore, an interesting line of research would be investigating whether epigenetic changes via ncRNA also regulate axonal regeneration. A few studies seem to indicate that it might be a possibility. For example, a mutual feedback loop between miR-138 and SIRT1 has been described, which contributes to axonal regeneration. Sirt1 is the target of miR-138 in DRG neurons, and in uninjured neurons the high level of miR-138 suppresses the expression of SIRT1, contributing to the low intrinsic axonal regeneration ability. Upon nerve injury, down-regulation of miR-138 correlates with an increase in Sirt1 levels, which is required for axonal regeneration and it also acts as a transcriptional repressor to directly suppress the expression of miR-138 [116]. As another example, miR-206 is upregulated in a mouse model of ALS, and upon nerve injury in wild type mice, where it is beneficial for axonal regeneration. It regulates HDAC4 via transcriptional repression, which might promote axonal regeneration as suggested by mice lacking HDAC4 that showed faster muscle reinnervation after axonal injury $[117,118]$.

mRNA post translational modifications, constituting the so called epitranscriptome, contribute to the regulation of gene expression as well. Among them, N-6-methyladenosine $\left(\mathrm{m}^{6} \mathrm{~A}\right)$ is the most abundant and has been implicated in mRNA stability and processing, export, and translation [119, 120]. Intriguingly, a recent report [121] has demonstrated that peripheral nerve injury is associated with an increased level of m6A in DRG neurons, with a peak at 1-3 days upon injury. Using $\mathrm{m}^{6} \mathrm{~A}$-SMART-seq the authors found that more than 6000 transcripts were $\mathrm{m}^{6} \mathrm{~A}$ tagged, and about 182 were upregulated at $24 \mathrm{~h}$ upon sciatic nerve injury. Among them, about 30 RAGs were identified, such as Atf3, Gadd45, Sox11, and Tet3. Interestingly, gene ontology analysis revealed enrichment of ribosomal and translation machinery proteins. Using $m^{6} \mathrm{~A}$-CLIP-SMART-seq, having single base resolution, the $\mathrm{m}^{6} \mathrm{~A}$ sites were found enriched in start sites, stop codons, and exons. Interestingly, RAG transcripts displayed the larger gain in $\mathrm{m} 6 \mathrm{~A}$, and in some of them new sites were found, mostly in exons. Neuronal deletion of Methyltransferase like 14 (Mttl14), the enzyme responsible for such modification, did not result in significant effect on gene expression in naïve or injury conditions, whereas it strongly impaired injury dependent de novo protein synthesis, in accordance with the proposed role of RNA $\mathrm{m}^{6} \mathrm{~A}$ [120]. This led to impaired nerve regeneration and functional recovery at the heat-evoked hind paw withdrawal test in vivo, without affecting neuronal death. A similar effect was observed upon the KO of the Ythd1 factor, a protein involved in the facilitation of translation of $\mathrm{m}^{6} \mathrm{~A}$ tagged mRNA [122]. More importantly, Mettl14 KD resulted also in impaired RGN survival and axonal elongation in a model of PTEN deletion-induced axonal regeneration upon ONC. Since Mttl14 KO did not affect global gene expression levels, and $\mathrm{m}^{6} \mathrm{~A}$ tagged transcripts were enriched for translation machinery proteins, this works raises the intriguing hypothesis that $\mathrm{m}^{6} \mathrm{~A}$ tagging promotes global protein synthesis upon injury required for sustained axonal regeneration. Therefore, the investigation of $\mathrm{m}^{6} \mathrm{~A}$ induced protein repertoires upon peripheral and central axonal injury represents a new promising field of investigation.

The key signalling pathways relying on epigenetic control of axonal regeneration described in this manuscript have been summarised in a diagramme (Figure 1). 


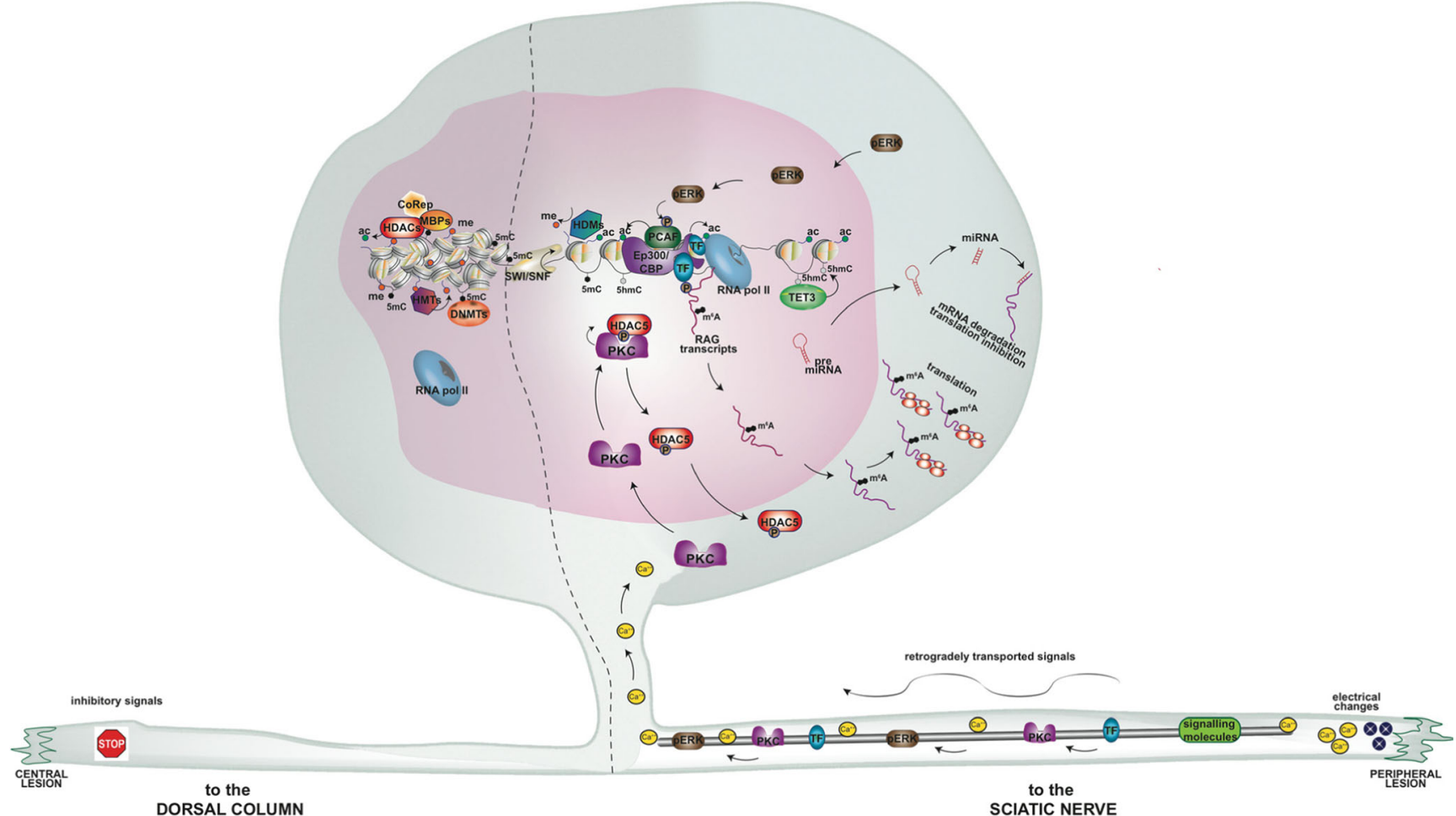

Fig. 1 Cartoon summarizing epigenetic pathways upon an axonal injury in the peripheral and central nervous system, based upon in vivo and in culture experimental evidence. A dorsal root ganglia neuron is schematized with a peripheral branch towards the sciatic nerve and a central branch towards the dorsal column. Upon a nerve injury (on the right) changes in electrical activity and $\mathrm{Ca}^{++}$influx result in activation of signalling molecules (ERK and PKC among them) and transcription factors (TFs), which are retrogradely transported to the soma. PKC leads to phosphorylation and nuclear export of histone deacetylase 5 (HDAC5), while pERK leads to phosphorylation and activation of p300/CBP associated factor (PCAF), resulting in increased histone and TF acetylation (ac) at the gene promoters of regeneration associated genes (RAGs). Calcium signalling is also responsible for the DNA

\section{Limitations and Further Perspectives}

So far, only limited regeneration has been achieved by $\mathrm{TF}$ overexpression, pharmacological or genetic modulation of epigenetic modifiers. The heterogeneity of the nervous system is a challenge to the investigation of neuronal vs non neuronal mechanisms and especially so in case of very rare biological events, such as in the case of DNA 5hmC. Moreover, very few systematic unbiased studies have directly compared and correlated transcriptional changes and epigenetic modifications specifically in a peripheral vs central axonal injury paradigm, in the attempt to identify which epigenetic modification/s might be able to reprogram CNS neuron in a regenerative state $[59,97,103,106]$. So far only DNA methylation and hydroxymethylation profiles have been systematically assessed, although DNA methylation was limited by the depth of the microarrays employed [59, 97, 103].

Moreover, at the moment, only histone acetylation and partially methylation have been investigated. However the hydroxymethylation $(5 \mathrm{hmC})$ mediated by Ten Eleven Translocation 3 (TET3) at the RAG gene bodies and enhancers. Chromatin remodelling factors, eg. SWItch/Sucrose NonFermentable (SWI/SNF), might also play a role. Altogether, this results in increased RAG transcription, via RNA polymerase II (RNA pol II). Some transcripts can be modified by $\mathrm{m}^{6} \mathrm{~A}$ addition, leading to increased translation efficiency. On the contrary, HDAC transcripts can be miRNA targets, representing positive feedbacks for sustained regeneration. Upon a dorsal column injury (on the left part) activation of inhibitory signalling pathways does not allow epigenetic remodelling. DNA methylation $(5 \mathrm{mC})$ and histone methylation (me) recruit silencing factors, eg. Methyl binding proteins (MDMs), histone deacetylase (HDACs), histone methyltransferase (HMTs), and nuclear corepressor (CoRep)

histone code is much richer, including phosphorylation, ubiquitinylation, SUMOylation, ADP ribosylation, and deamination [35]. Moreover, modifications of the histone globular domains have been described, associated to nucleosome assembly and transcription regulation [123]. Interestingly, lysine acylation, including propionylation, butyrylation, succinylation, malonylation, crotonylation, and glutarylation has been recently described to be associated to either globular or tail domain [123]. Acyl-coA groups used for these modifications derive from metabolic reactions, such as fatty acid $\beta$ oxidation, citrate cycle, and the aminoacid metabolism, suggesting a metabolic control of the epigenome. Since metabolic responses upon brain injury have been described [124], it would be interesting to assess whether metabolic changes might affect axonal regeneration via epigenetic remodelling. Next, in addition to HPTM, histone turnover of the highly dynamic $\mathrm{H} 3.3$ histone variant has been associated to neuronal transcription and plasticity [125], and the role in axonal regeneration remains unknown. 
Chromatin high-order structure, determined by the action of chromatin remodellers and architectural proteins, is an important feature in gene expression regulation $[27,126]$. Chromatin remodellers, belonging to the SWItch/Sucrose NonFermentable (SWI/SNF), Imitation SWI (ISWI), Nucleosome Remodelling and Deacetylase (NURD/Mi-2/CHD), and Inositol Auxotroph 80 (INO80) families, are motor proteins that use the energy of ATP hydrolysis to alter nucleosome structure, affecting transcription initiation and elongation, histone variant deposition, DNA repair [29-31]. Architectural proteins, such as CCCTC-binding factor (CTCF) and cohesin, play a major role in genome organization by their ability to assist in the formation of long-range contacts between genomic loci via chromatin loops, including between promoters and enhancers, facilitating transcription [127-129]. Although changes in chromatin structure have been involved in several aspects of neuronal biology and pathology, such as neurogenesis, neuronal differentiation and maturation, dendrite morphogenesis and growth, cognition, neurodevelopmental and neurodegenerative disorders [127, 130-136], it is not known if they might play a role in axonal regeneration.

Genome wide extensive studies, such as Chromatin immunoprecipitation (ChIP)-, Assay for transposase accessible chromatin (ATAC)-, micrococcal nuclease (MNase)-, bisulfite (BS), Hi capture sequencing are necessary to complete the picture of the epigenetic modifications, the chromatin conformation and structural changes occurring upon axonal injury, and to understand how all these cooperate in driving or hindering axonal regeneration. The epigenetic studies discussed so far have the limitations of reflecting a mixed neuronal, glial and at times immune cell populations of cells. Performing these assessments in specifically enriched cell populations will be fundamental to dissect the contribution of neurons vs glial or infiltrating immune cells to axonal regeneration. A further refinement would be the use of single cell epigenome sequencing technologies [137] that will address possible neuronal type specific differences in axonal growth potential.

Ultimately, these studies could pave the way to the ambitious goal to reprogram neurons into a regenerative state, in a timely controlled and specific manner, taking advantage for example of novel tools such as CRISPR/Cas9 genome editing.

\begin{abstract}
Acknowledgements We would like to thank start up funds-Division of Brain Sciences, Imperial College London (SDG), Wings for Life (SDG); the Henry Smith Charity (SDG), Rosetrees Trust (SDG), the Leverhulme Trust (SDG), ISRT (SDG). The research was supported by the National Institute for Health Research (NIHR) Imperial Biomedical Research Centre (SDG). The views expressed are those of the author(s) and not necessarily those of the NHS, the NIHR or the Department of Health.
\end{abstract}

Required Author Forms Disclosure forms provided by the authors are available with the online version of this article.
Open Access This article is distributed under the terms of the Creative Commons Attribution 4.0 International License (http:// creativecommons.org/licenses/by/4.0/), which permits unrestricted use, distribution, and reproduction in any medium, provided you give appropriate credit to the original author(s) and the source, provide a link to the Creative Commons license, and indicate if changes were made.

\section{References}

1. Liu K, Tedeschi A, Park KK, He Z. Neuronal intrinsic mechanisms of axon regeneration. Annual review of neuroscience 2011;34:131-152.

2. Ferguson TA, Son YJ. Extrinsic and intrinsic determinants of nerve regeneration. Journal of tissue engineering 2011;2: 2041731411418392.

3. Afshari FT, Kappagantula S, Fawcett JW. Extrinsic and intrinsic factors controlling axonal regeneration after spinal cord injury. Expert reviews in molecular medicine 2009; 11:e37.

4. Silver J, Miller JH. Regeneration beyond the glial scar. Nature reviews Neuroscience 2004;5:146-156.

5. David S, Aguayo AJ. Axonal elongation into peripheral nervous system "bridges" after central nervous system injury in adult rats. Science 1981;214:931-933.

6. Yiu G, He Z. Glial inhibition of CNS axon regeneration. Nature reviews Neuroscience 2006;7:617-627.

7. Lee JK, Kim JE, Sivula M, Strittmatter SM. Nogo receptor antagonism promotes stroke recovery by enhancing axonal plasticity. The Journal of neuroscience : the official journal of the Society for Neuroscience 2004;24:6209-6217.

8. Bradbury EJ, Moon LD, Popat RJ, et al. Chondroitinase ABC promotes functional recovery after spinal cord injury. Nature 2002;416:636-640.

9. Schnell L, Schwab ME. Axonal regeneration in the rat spinal cord produced by an antibody against myelin-associated neurite growth inhibitors. Nature 1990;343:269-272.

10. Spaulding EL, Burgess RW. Accumulating Evidence for Axonal Translation in Neuronal Homeostasis. Frontiers in neuroscience 2017; 11:312.

11. Gumy LF, Tan CL, Fawcett JW. The role of local protein synthesis and degradation in axon regeneration. Experimental neurology 2010;223:28-37.

12. Tedeschi A. Tuning the orchestra: transcriptional pathways controlling axon regeneration. Frontiers in molecular neuroscience 2011;4:60

13. Verdu E, Buti M, Navarro X. The effect of aging on efferent nerve fibers regeneration in mice. Brain research 1995;696:76-82.

14. Verdu E, Ceballos D, Vilches JJ, Navarro X. Influence of aging on peripheral nerve function and regeneration. Journal of the peripheral nervous system : JPNS 2000;5:191-208.

15. Goldberg JL, Klassen MP, Hua Y, Barres BA. Amacrine-signaled loss of intrinsic axon growth ability by retinal ganglion cells. Science 2002;296:1860-1864.

16. Blackmore M, Letourneau PC. Changes within maturing neurons limit axonal regeneration in the developing spinal cord. Journal of neurobiology 2006;66:348-360.

17. Geoffroy CG, Hilton BJ, Tetzlaff W, Zheng B. Evidence for an AgeDependent Decline in Axon Regeneration in the Adult Mammalian Central Nervous System. Cell reports 2016;15:238-246.

18. Wang JT, Kunzevitzky NJ, Dugas JC, Cameron M, Barres BA, Goldberg JL. Disease gene candidates revealed by expression profiling of retinal ganglion cell development. The Journal of neuroscience : the official journal of the Society for Neuroscience 2007;27:8593-8603. 
19. Venkatesh I, Simpson MT, Coley DM, Blackmore MG. Epigenetic profiling reveals a developmental decrease in promoter accessibility during cortical maturation in vivo. Neuroepigenetics 2016;8:19-26.

20. Ma TC, Willis DE. What makes a RAG regeneration associated? Frontiers in molecular neuroscience 2015;8:43.

21. Fagoe ND, Attwell CL, Kouwenhoven D, Verhaagen J, Mason MR. Overexpression of ATF3 or the combination of ATF3, cJun, STAT3 and Smad1 promotes regeneration of the central axon branch of sensory neurons but without synergistic effects. Human molecular genetics 2015;24:6788-6800.

22. Venkatesh I, Blackmore MG. Selecting optimal combinations of transcription factors to promote axon regeneration: Why mechanisms matter. Neuroscience letters 2017;652:64-73.

23. Sun F, Park KK, Belin S, et al. Sustained axon regeneration induced by co-deletion of PTEN and SOCS3. Nature 2011;480:372375 .

24. Gaub P, Tedeschi A, Puttagunta R, Nguyen T, Schmandke A, Di Giovanni S. HDAC inhibition promotes neuronal outgrowth and counteracts growth cone collapse through $\mathrm{CBP} / \mathrm{p} 300$ and $\mathrm{P} / \mathrm{CAF}$ dependent $\mathrm{p} 53$ acetylation. Cell death and differentiation 2010;17: 1392-1408.

25. Gaub P, Joshi Y, Wuttke A, et al. The histone acetyltransferase p300 promotes intrinsic axonal regeneration. Brain : a journal of neurology 2011;134:2134-2148.

26. Luger K, Dechassa ML, Tremethick DJ. New insights into nucleosome and chromatin structure: an ordered state or a disordered affair? Nature reviews. Molecular cell biology 2012;13:436-447.

27. Woodcock CL, Ghosh RP. Chromatin higher-order structure and dynamics. Cold Spring Harbor perspectives in biology 2010;2: a000596.

28. $\mathrm{Hu} \mathrm{Z}$, Tee WW. Enhancers and chromatin structures: regulatory hubs in gene expression and diseases. Bioscience reports 2017;37.

29. Geiman TM, Robertson KD. Chromatin remodeling, histone modifications, and DNA methylation-how does it all fit together? Journal of cellular biochemistry 2002;87:117-125.

30. Vignali M, Hassan AH, Neely KE, Workman JL. ATP-dependent chromatin-remodeling complexes. Molecular and cellular biology 2000;20:1899-1910.

31. Li E. Chromatin modification and epigenetic reprogramming in mammalian development. Nature reviews Genetics 2002;3:662673.

32. Hergeth SP, Schneider R. The H1 linker histones: multifunctional proteins beyond the nucleosomal core particle. EMBO reports 2015;16:1439-1453.

33. Wasylyk B, Chambon P. Transcription by eukaryotic RNA polymerases A and B of chromatin assembled in vitro. European journal of biochemistry 1979;98:317-327.

34. Luger K, Mader AW, Richmond RK, Sargent DF, Richmond TJ. Crystal structure of the nucleosome core particle at $2.8 \mathrm{~A}$ resolution. Nature 1997;389:251-260.

35. Kouzarides T. Chromatin modifications and their function. Cell 2007;128:693-705.

36. Haberland M, Montgomery RL, Olson EN. The many roles of histone deacetylases in development and physiology: implications for disease and therapy. Nature reviews. Genetics 2009;10:32-42.

37. Shahbazian MD, Grunstein M. Functions of site-specific histone acetylation and deacetylation. Annual review of biochemistry 2007;76:75-100

38. Fischle W, Dequiedt F, Hendzel MJ, et al. Enzymatic activity associated with class II HDACs is dependent on a multiprotein complex containing HDAC3 and SMRT/N-CoR. Molecular cell 2002;9:45-57.

39. Robertson KD. DNA methylation and human disease. Nature reviews Genetics 2005;6:597-610.

40. Bestor TH. The DNA methyltransferases of mammals. Human molecular genetics 2000;9:2395-2402.
41. Probst AV, Dunleavy E, Almouzni G. Epigenetic inheritance during the cell cycle. Nature reviews Molecular cell biology 2009;10: 192-206.

42. Okano M, Bell DW, Haber DA, Li E. DNA methyltransferases Dnmt3a and Dnmt3b are essential for de novo methylation and mammalian development. Cell 1999;99:247-257.

43. Lister R, Pelizzola M, Dowen RH, et al. Human DNA methylomes at base resolution show widespread epigenomic differences. Nature 2009;462:315-322.

44. Jang HS, Shin WJ, Lee JE, Do JT. CpG and Non-CpG Methylation in Epigenetic Gene Regulation and Brain Function. Genes 2017;8.

45. Deaton AM, Bird A. CpG islands and the regulation of transcription. Genes \& development 2011;25:1010-1022.

46. Bird AP. CpG-rich islands and the function of DNA methylation. Nature 1986;321:209-213.

47. Wade PA. Methyl CpG-binding proteins and transcriptional repression. BioEssays : news and reviews in molecular, cellular and developmental biology 2001;23:1131-1137.

48. Clouaire T, Stancheva I. Methyl-CpG binding proteins: specialized transcriptional repressors or structural components of chromatin? Cellular and molecular life sciences : CMLS 2008;65: 1509-1522.

49. Rasmussen KD, Helin K. Role of TET enzymes in DNA methylation, development, and cancer. Genes \& development 2016;30: 733-750.

50. Tahiliani M, Koh KP, Shen Y, et al. Conversion of 5methylcytosine to 5-hydroxymethylcytosine in mammalian DNA by MLL partner TET1. Science 2009;324:930-935.

51. Kohli RM, Zhang Y. TET enzymes, TDG and the dynamics of DNA demethylation. Nature 2013;502:472-479.

52. Ito S, Shen L, Dai Q, et al. Tet proteins can convert 5methylcytosine to 5-formylcytosine and 5-carboxylcytosine. Science 2011;333:1300-1303.

53. Branco MR, Ficz G, Reik W. Uncovering the role of 5hydroxymethylcytosine in the epigenome. Nature reviews Genetics 2011;13:7-13.

54. Khare T, Pai S, Koncevicius K, et al. 5-hmC in the brain is abundant in synaptic genes and shows differences at the exon-intron boundary. Nature structural \& molecular biology 2012;19:10371043.

55. Lv L, Sun Y, Han X, Xu CC, Tang YP, Dong Q. Valproic acid improves outcome after rodent spinal cord injury: potential roles of histone deacetylase inhibition. Brain research 2011;1396:60-68.

56. Lv L, Han X, Sun Y, Wang X, Dong Q. Valproic acid improves locomotion in vivo after SCI and axonal growth of neurons in vitro. Experimental neurology 2012;233:783-790.

57. Finelli MJ, Wong JK, Zou H. Epigenetic regulation of sensory axon regeneration after spinal cord injury. The Journal of neuroscience : the official journal of the Society for Neuroscience 2013;33:19664-19676.

58. Neumann S, Woolf CJ. Regeneration of dorsal column fibers into and beyond the lesion site following adult spinal cord injury. Neuron 1999;23:83-91.

59. Puttagunta R, Tedeschi A, Soria MG, et al. PCAF-dependent epigenetic changes promote axonal regeneration in the central nervous system. Nature communications 2014;5:3527.

60. van Kesteren RE, Mason MR, Macgillavry HD, Smit AB, Verhaagen J. A gene network perspective on axonal regeneration. Frontiers in molecular neuroscience 2011;4:46.

61. Hanz S, Fainzilber M. Integration of retrograde axonal and nuclear transport mechanisms in neurons: implications for therapeutics. The Neuroscientist : a review journal bringing neurobiology, neurology and psychiatry 2004;10:404-408.

62. Rishal I, Fainzilber M. Axon-soma communication in neuronal injury. Nature reviews Neuroscience 2014;15:32-42. 
63. Wolf JA, Stys PK, Lusardi T, Meaney D, Smith DH. Traumatic axonal injury induces calcium influx modulated by tetrodotoxinsensitive sodium channels. The Journal of neuroscience : the official journal of the Society for Neuroscience 2001;21:1923-1930.

64. Mandolesi G, Madeddu F, Bozzi Y, Maffei L, Ratto GM. Acute physiological response of mammalian central neurons to axotomy: ionic regulation and electrical activity. FASEB journal : official publication of the Federation of American Societies for Experimental Biology 2004;18:1934-1936.

65. Makwana M, Raivich G. Molecular mechanisms in successful peripheral regeneration. The FEBS journal 2005;272:2628-2638.

66. Abe N, Cavalli V. Nerve injury signaling. Current opinion in neurobiology 2008;18:276-283.

67. Chen ZL, Yu WM, Strickland S. Peripheral regeneration. Annual review of neuroscience 2007;30:209-233.

68. Shin JE, Cho Y, Beirowski B, Milbrandt J, Cavalli V, DiAntonio A. Dual leucine zipper kinase is required for retrograde injury signaling and axonal regeneration. Neuron 2012;74:1015-1022.

69. Cho Y, Cavalli V. HDAC5 is a novel injury-regulated tubulin deacetylase controlling axon regeneration. The EMBO journal 2012;31:3063-3078.

70. Cho Y, Sloutsky R, Naegle KM, Cavalli V. Injury-induced HDAC5 nuclear export is essential for axon regeneration. Cell 2013;155:894-908.

71. Grozinger CM, Hassig CA, Schreiber SL. Three proteins define a class of human histone deacetylases related to yeast Hdalp. Proceedings of the National Academy of Sciences of the United States of America 1999;96:4868-4873.

72. Pelzel HR, Schlamp CL, Nickells RW. Histone H4 deacetylation plays a critical role in early gene silencing during neuronal apoptosis. BMC neuroscience 2010;11:62.

73. He X, Zhang L, Queme LF, et al. A histone deacetylase 3dependent pathway delimits peripheral myelin growth and functional regeneration. Nature medicine 2018.

74. Kuboyama T, Wahane S, Huang Y, et al. HDAC3 inhibition ameliorates spinal cord injury by immunomodulation. Scientific reports 2017;7:8641.

75. Schmitt HM, Pelzel HR, Schlamp CL, Nickells RW. Histone deacetylase 3 (HDAC3) plays an important role in retinal ganglion cell death after acute optic nerve injury. Molecular neurodegeneration 2014;9:39.

76. Schmitt HM, Schlamp CL, Nickells RW. Targeting HDAC3 Activity with RGFP966 Protects Against Retinal Ganglion Cell Nuclear Atrophy and Apoptosis After Optic Nerve Injury. Journal of ocular pharmacology and therapeutics : the official journal of the Association for Ocular Pharmacology and Therapeutics 2017.

77. Lebrun-Julien F, Suter U. Combined HDAC1 and HDAC2 Depletion Promotes Retinal Ganglion Cell Survival After Injury Through Reduction of p53 Target Gene Expression. ASN neuro 2015;7.

78. Lin YH, Dong J, Tang Y, et al. Opening a New Time Window for Treatment of Stroke by Targeting HDAC2. The Journal of neuroscience : the official journal of the Society for Neuroscience 2017;37:6712-6728.

79. Tang Y, Lin YH, Ni HY, et al. Inhibiting Histone Deacetylase 2 (HDAC2) Promotes Functional Recovery From Stroke. Journal of the American Heart Association 2017;6.

80. Singh BN, Zhang G, Hwa YL, Li J, Dowdy SC, Jiang SW. Nonhistone protein acetylation as cancer therapy targets. Expert review of anticancer therapy 2010;10:935-954.

81. Tedeschi A, Nguyen T, Puttagunta R, Gaub P, Di Giovanni S. A p53-CBP/p300 transcription module is required for GAP-43 expression, axon outgrowth, and regeneration. Cell death and differentiation 2009;16:543-554.

82. Joshi Y, Soria MG, Quadrato G, et al. The MDM4/MDM2-p53IGF1 axis controls axonal regeneration, sprouting and functional recovery after CNS injury. Brain : a journal of neurology 2015;138:1843-1862.

83. Zou H, Ho C, Wong K, Tessier-Lavigne M. Axotomy-induced Smadl activation promotes axonal growth in adult sensory neurons. The Journal of neuroscience : the official journal of the Society for Neuroscience 2009;29:7116-7123.

84. Inoue $\mathrm{Y}$, Itoh $\mathrm{Y}$, Abe $\mathrm{K}$, et al. Smad3 is acetylated by $\mathrm{p} 300 / \mathrm{CBP}$ to regulate its transactivation activity. Oncogene 2007;26:500-508.

85. Rivieccio MA, Brochier C, Willis DE, et al. HDAC6 is a target for protection and regeneration following injury in the nervous system. Proceedings of the National Academy of Sciences of the United States of America 2009;106:19599-19604.

86. Zhang X, Yuan Z, Zhang Y, et al. HDAC6 modulates cell motility by altering the acetylation level of cortactin. Molecular cell 2007;27:197-213.

87. Lowery LA, Van Vactor D. The trip of the tip: understanding the growth cone machinery. Nature reviews Molecular cell biology 2009;10:332-343.

88. Yuan ZL, Guan YJ, Chatterjee D, Chin YE. Stat3 dimerization regulated by reversible acetylation of a single lysine residue. Science 2005;307:269-273.

89. Faiola F, Liu X, Lo S, et al. Dual regulation of c-Myc by p300 via acetylation-dependent control of Myc protein turnover and coactivation of Myc-induced transcription. Molecular and cellular biology 2005;25:10220-10234.

90. Iskandar BJ, Rizk E, Meier B, et al. Folate regulation of axonal regeneration in the rodent central nervous system through DNA methylation. The Journal of clinical investigation 2010;120:16031616.

91. Crider KS, Yang TP, Berry RJ, Bailey LB. Folate and DNA methylation: a review of molecular mechanisms and the evidence for folate's role. Advances in nutrition 2012;3:21-38.

92. Ghoshal K, Li X, Datta J, et al. A folate- and methyl-deficient diet alters the expression of DNA methyltransferases and methyl $\mathrm{CpG}$ binding proteins involved in epigenetic gene silencing in livers of F344 rats. The Journal of nutrition 2006;136:1522-1527.

93. Pollema-Mays SL, Centeno MV, Apkarian AV, Martina M. Expression of DNA methyltransferases in adult dorsal root ganglia is cell-type specific and up regulated in a rodent model of neuropathic pain. Frontiers in cellular neuroscience 2014;8:217.

94. Kadriu B, Guidotti A, Chen Y, Grayson DR. DNA methyltransferases1 (DNMT1) and 3a (DNMT3a) colocalize with GAD67positive neurons in the GAD67-GFP mouse brain. The Journal of comparative neurology 2012;520:1951-1964.

95. Inano K, Suetake I, Ueda T, et al. Maintenance-type DNA methyltransferase is highly expressed in post-mitotic neurons and localized in the cytoplasmic compartment. Journal of biochemistry 2000;128:315-321.

96. Feng J, Chang H, Li E, Fan G. Dynamic expression of de novo DNA methyltransferases Dnmt3a and Dnmt3b in the central nervous system. Journal of neuroscience research 2005;79:734-746.

97. Lindner R, Puttagunta R, Nguyen T, Di Giovanni S. DNA methylation temporal profiling following peripheral versus central nervous system axotomy. Scientific data 2014;1:140038.

98. Tedeschi A, Dupraz S, Laskowski CJ, et al. The Calcium Channel Subunit Alpha2delta2 Suppresses Axon Regeneration in the Adult CNS. Neuron 2016;92:419-434.

99. Chen D, Yu SP, Wei L. Ion channels in regulation of neuronal regenerative activities. Translational stroke research 2014;5:156162.

100. Li S, Xue C, Yuan Y, et al. The transcriptional landscape of dorsal root ganglia after sciatic nerve transection. Scientific reports 2015;5:16888.

101. Hu G, Huang K, Hu Y, et al. Single-cell RNA-seq reveals distinct injury responses in different types of DRG sensory neurons. Scientific reports 2016;6:31851. 
102. Weng YL, An R, Cassin J, et al. An Intrinsic Epigenetic Barrier for Functional Axon Regeneration. Neuron 2017;94:337-346 e336.

103. Loh YE, Koemeter-Cox A, Finelli MJ, Shen L, Friedel RH, Zou H. Comprehensive mapping of 5-hydroxymethylcytosine epigenetic dynamics in axon regeneration. Epigenetics 2017;12:77-92.

104. Cho Y, Shin JE, Ewan EE, Oh YM, Pita-Thomas W, Cavalli V. Activating Injury-Responsive Genes with Hypoxia Enhances Axon Regeneration through Neuronal HIF-1alpha. Neuron 2015;88:720-734.

105. Schwaiger FW, Hager G, Schmitt AB, et al. Peripheral but not central axotomy induces changes in Janus kinases (JAK) and signal transducers and activators of transcription (STAT). The European journal of neuroscience 2000;12:1165-1176.

106. Chandran V, Coppola G, Nawabi H, et al. A Systems-Level Analysis of the Peripheral Nerve Intrinsic Axonal Growth Program. Neuron 2016;89:956-970.

107. Lin D, Ippolito GC, Zong RT, Bryant J, Koslovsky J, Tucker P. Bright/ARID3A contributes to chromatin accessibility of the immunoglobulin heavy chain enhancer. Molecular cancer 2007;6:23.

108. Riedel CG, Dowen RH, Lourenco GF, et al. DAF-16 employs the chromatin remodeller SWI/SNF to promote stress resistance and longevity. Nature cell biology 2013;15:491-501.

109. Zaret KS, Carroll JS. Pioneer transcription factors: establishing competence for gene expression. Genes \& development 2011;25:2227-2241.

110. Liu K, Lu Y, Lee JK, et al. PTEN deletion enhances the regenerative ability of adult corticospinal neurons. Nature neuroscience 2010;13:1075-1081.

111. Tan AY, Manley JL. The TET family of proteins: functions and roles in disease. Journal of molecular cell biology 2009;1:82-92.

112. Bauer C, Gobel K, Nagaraj N, et al. Phosphorylation of TET proteins is regulated via O-GlcNAcylation by the O-linked Nacetylglucosamine transferase (OGT). The Journal of biological chemistry 2015;290:4801-4812.

113. Dehennaut V, Leprince D, Lefebvre T. O-GlcNAcylation, an Epigenetic Mark. Focus on the Histone Code, TET Family Proteins, and Polycomb Group Proteins. Frontiers in endocrinology 2014;5:155.

114. Peschansky VJ, Wahlestedt C. Non-coding RNAs as direct and indirect modulators of epigenetic regulation. Epigenetics 2014;9: 3-12.

115. Zhou S, Ding F, Gu X. Non-coding RNAs as Emerging Regulators of Neural Injury Responses and Regeneration. Neuroscience bulletin 2016;32:253-264.

116. Liu CM, Wang RY, Saijilafu, Jiao ZX, Zhang BY, Zhou FQ. MicroRNA-138 and SIRT1 form a mutual negative feedback loop to regulate mammalian axon regeneration. Genes \& development 2013;27:1473-1483

117. Cohen TJ, Waddell DS, Barrientos T, et al. The histone deacetylase HDAC4 connects neural activity to muscle transcriptional reprogramming. The Journal of biological chemistry 2007;282:33752-33759.

118. Williams AH, Valdez G, Moresi V, et al. MicroRNA-206 delays ALS progression and promotes regeneration of neuromuscular synapses in mice. Science 2009;326:1549-1554.

119. Roundtree IA, Evans ME, Pan T, He C. Dynamic RNA Modifications in Gene Expression Regulation. Cell 2017;169: 1187-1200.
120. Zhao BS, Roundtree IA, He C. Post-transcriptional gene regulation by mRNA modifications. Nature reviews Molecular cell biology 2017;18:31-42.

121. Weng YL, Wang X, An R, et al. Epitranscriptomic m(6)A Regulation of Axon Regeneration in the Adult Mammalian Nervous System. Neuron 2018;97:313-325 e316.

122. Shi H, Wang X, Lu Z, et al. YTHDF3 facilitates translation and decay of N(6)-methyladenosine-modified RNA. Cell research 2017;27:315-328.

123. Kebede AF, Schneider R, Daujat S. Novel types and sites of histone modifications emerge as players in the transcriptional regulation contest. The FEBS journal 2015;282:1658-1674.

124. Bartnik-Olson BL, Harris NG, Shijo K, Sutton RL. Insights into the metabolic response to traumatic brain injury as revealed by (13)C NMR spectroscopy. Frontiers in neuroenergetics 2013;5:8.

125. Maze I, Wenderski W, Noh KM, et al. Critical Role of Histone Turnover in Neuronal Transcription and Plasticity. Neuron 2015;87:77-94.

126. Li G, Reinberg D. Chromatin higher-order structures and gene regulation. Current opinion in genetics \& development 2011;21: 175-186.

127. Beagan JA, Duong MT, Titus KR, et al. YY1 and CTCF orchestrate a 3D chromatin looping switch during early neural lineage commitment. Genome research 2017;27:1139-1152.

128. Zuin J, Dixon JR, van der Reijden MI, et al. Cohesin and CTCF differentially affect chromatin architecture and gene expression in human cells. Proceedings of the National Academy of Sciences of the United States of America 2014;111:996-1001.

129. Cubenas-Potts C, Corces VG. Architectural proteins, transcription, and the three-dimensional organization of the genome. FEBS letters 2015;589:2923-2930.

130. Nitarska J, Smith JG, Sherlock WT, et al. A Functional Switch of NuRD Chromatin Remodeling Complex Subunits Regulates Mouse Cortical Development. Cell reports 2016;17:1683-1698.

131. Sokpor G, Xie Y, Rosenbusch J, Tuoc T. Chromatin Remodeling BAF (SWI/SNF) Complexes in Neural Development and Disorders. Frontiers in molecular neuroscience 2017;10:243.

132. Berson A, Sartoris A, Nativio R, et al. TDP-43 Promotes Neurodegeneration by Impairing Chromatin Remodeling. Current biology : CB 2017;27:3579-3590 e3576.

133. Tibshirani M, Zhao B, Gentil BJ, et al. Dysregulation of chromatin remodelling complexes in amyotrophic lateral sclerosis. Human molecular genetics 2017;26:4142-4152.

134. Wu JI, Lessard J, Olave IA, et al. Regulation of dendritic development by neuron-specific chromatin remodeling complexes. Neuron 2007;56:94-108.

135. Medrano-Fernandez A, Barco A. Nuclear organization and 3D chromatin architecture in cognition and neuropsychiatric disorders. Molecular brain 2016;9:83.

136. Bonev B, Mendelson Cohen N, Szabo Q, et al. Multiscale 3D Genome Rewiring during Mouse Neural Development. Cell 2017;171:557-572 e524.

137. Wen L, Tang F. Single cell epigenome sequencing technologies. Molecular aspects of medicine 2018;59:62-69. 